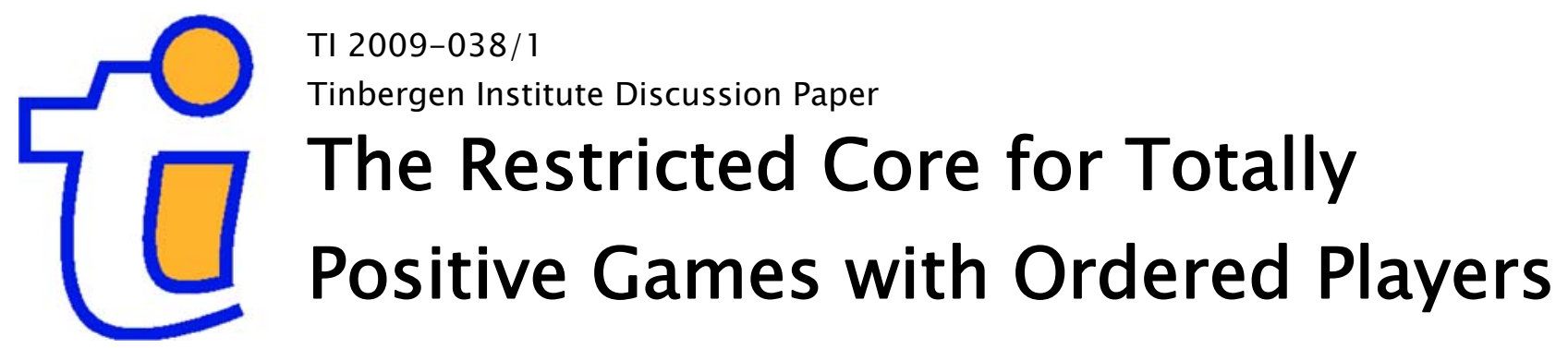

René van den Brink ${ }^{1}$

Gerard van der Laan'

Valeri Vasil'ev²

1 Department of Econometrics, VU University Amsterdam, and Tinbergen Institute, The Netherlands;

2 Sobolev Institute of Mathematics, Novosibirsk, Russia. 


\section{Tinbergen Institute}

The Tinbergen Institute is the institute for economic research of the Erasmus Universiteit Rotterdam, Universiteit van Amsterdam, and Vrije Universiteit Amsterdam.

Tinbergen Institute Amsterdam

Roetersstraat 31

1018 WB Amsterdam

The Netherlands

Tel.: +31(0)205513500

Fax: $+31(0) 205513555$

Tinbergen Institute Rotterdam

Burg. Oudlaan 50

3062 PA Rotterdam

The Netherlands

Tel.: + $31(0) 104088900$

Fax: $+31(0) 104089031$

Most TI discussion papers can be downloaded at http://www.tinbergen.nl. 


\title{
The Restricted Core for totally positive games with ordered players ${ }^{1}$
}

\author{
René van den Brink ${ }^{2}$ Gerard van der Laan ${ }^{3}$ Valeri Vasil'ev ${ }^{4}$
}

April 29, 2009

\footnotetext{
${ }^{1}$ This research has been done while the third author was visiting the Tinbergen Institute, VU University Amsterdam, on NWO-grant 047.017.017 within the framework of Dutch-Russian cooperation. This author would like to appreciate also financial support from the Russian Leading Scientific Schools Fund (grant 4113.2008.6) and Russian Fund for Basic Research (grant 07-0600363a).

${ }^{2}$ J.R. van den Brink, Department of Econometrics and Tinbergen Institute, VU University, De Boelelaan 1105, 1081 HV Amsterdam, The Netherlands. E-mail: jrbrink@feweb.vu.nl

${ }^{3}$ G. van der Laan, Department of Econometrics and Tinbergen Institute, VU University, De Boelelaan 1105, $1081 \mathrm{HV}$ Amsterdam, The Netherlands. E-mail: glaan@feweb.vu.nl

${ }^{4}$ V.A. Vasil'ev, Sobolev Institute of Mathematics, Prosp. Koptyuga 4, 630090 Novosibirsk, Russia, E-mail: vasilev@math.nsc.ru
} 


\begin{abstract}
Recently, applications of cooperative game theory to economic allocation problems have gained popularity. In many such allocation problems, such as river games, queueing games and auction games, the game is totally positive (i.e., all dividends are nonnegative), and there is some hierarchical ordering of the players. In this paper we introduce the Restricted Core for such games with ordered players which is based on the distribution of dividends taking into account the hierarchical ordering of the players. For totally positive games this solution is always contained in the Core, and contains the well-known Shapley value (being the single-valued solution distributing the dividends equally among the players in the corresponding coalitions). For special orderings it equals the Core, respectively Shapley value. We provide an axiomatization and apply this solution to river games.
\end{abstract}

Keywords: Totally positive TU-game, Harsanyi dividends, Core, Shapley value, Harsanyi set, Selectope, Digraph, River game.

JEL code: C71 (Cooperative games) 


\section{Introduction}

A situation in which a finite set of players can obtain certain payoffs by cooperation can be described by a cooperative game with transferable utility, or simply a TU-game, which assigns to every subset of players or coalition a worth that these players can earn by cooperation. A solution for TU-games assigns a set of payoff vectors (possibly empty or consisting of a unique element) to every TU-game. In a payoff vector provided by a solution, the payoff assigned to a particular player depends on the payoffs that can be obtained by any coalition of players. The best-known single-valued solution is the Shapley value (Shapley, 1953), the best-known set-valued solution is the Core (Gillies, 1953).

In many economic allocation problems there is some hierarchical ordering of the players. For example, in the water allocation problem of Ambec and Sprumont (2002) agents are located along a river from upstream to downstream, in queueing games as considered in Maniquet (2003) the players can be ordered by their waiting cost, in sequencing situations as considered in, e.g. Curiel et al. (1989) the players (jobs) are ordered in an initial queue, in the auction situations of Graham et al. (1990) the agents can be ordered by their valuations of the good to be auctioned, and in the airport game of Littlechild and Owen (1973) the airplanes can be ordered by the cost of the landing strip necessary to build for these airplanes. Therefore, in this paper we assume that the players in a TUgame are part of some hierarchical structure that is represented by a directed graph. In such games with ordered players the payoff assigned to a player may depend on both the worths of the coalitions as well as the position of the player in the graph. In van den Brink et al. (2006) a solution for such games with ordered players is introduced that is based on distributing the Harsanyi dividends (see Harsanyi, 1959) of a game in the spirit of the Harsanyi set or Selectope. The Harsanyi set, see Vasil'ev $(1978,1981)$ and Vasil'ev and van der Laan (2002), assigns to every TU-game all Harsanyi payoff vectors, being those payoff vectors that are obtained by distributing every dividend in any possible way among the players in the corresponding coalition. An alternative definition of this set is given by the Selectope of a TU-game, see Hammer et al. (1977) and Derks et al. (2000), defined as the convex hull of all selectope vectors, where the selectope vectors are those vectors where every dividend is fully assigned to one player of the corresponding coalition. In case all dividends are nonnegative the Harsanyi set coincides with the Core of the game and thus consists of all efficient, group stable payoff vectors.

For games with ordered players, van den Brink et al. (2006) modified the Harsanyi set by requiring that for any coalition and for any two players $i$ and $j$ within a coalition, player $i$ gets a share in the dividend of the coalition that is at least as high as the share of player $j$, if player $i$ is a superior of $j$ in the ordering of the players. Obviously, because this puts restrictions on the distributions of the dividends, for every game with ordered players 
this modified Harsanyi set is a subset of the Harsanyi set of the game without ordering on the players. In case all dividends are nonnegative it follows that this modified Harsanyi set yields a refinement of the Core. Therefore, on this class of games with ordered players we refer to this modified Harsanyi set as the Restricted Core. For this class of games the Restricted Core is non-empty and generalizes both the Core and the Shapley value. In particular, for any directed graph the Shapley value of the game (without taking account of the ordering on the players) belongs to the Restricted Core, and it is the unique element of this set if the ordering is complete, i.e., every player dominates every other player in the sense that in the directed graph representing the ordering there is a directed arc from each player $i$ to every other player $j$. On the other hand, the Restricted Core is a subset of the Core of the game (without ordered players), and it equals the Core in case the directed graph representing the ordering is empty, i.e. no player dominates any other player.

For the class of games with ordered players and nonnegative dividends, we provide a characterization of the Restricted Core solution as the maximal (with respect to set inclusion) solution that satisfies the well-known efficiency, null player, additivity and nonnegativity properties, and a structural monotonicity property which reflects the hierarchical aspect of games with ordered players. By adding three more axioms, namely nonemptyness, convexity and a consistency property, we obtain a full axiomatization with eight logically independent axioms.

We argue that the Restricted Core yields a reasonable set of payoff vectors for economic allocation problems that have an underlying ordering of the players. For example, van den Brink et al. (2007) show that the solution proposed by Ambec and Sprumont (2002) for sharing the water in a river among the agents that are located along that river from upstream to downstream, is counterintuitive since it allocates the nonnegative dividends of cooperation fully to the most downstream agents in the corresponding coalitions, while the upstream players have control over the water flow. Applying the Restricted Core on the ordering from upstream to downstream allocates these dividends in such a way that upstream agents get a share that is at least equal to the share of downstream agents in the dividends ${ }^{1}$.

The paper is organized as follows. In Section 2 we discuss some preliminaries on TUgames and define the class of games with ordered players. In Section 3 we discuss the water distribution problem of Ambec and Sprumont (2002) as a motivating example. In Section 4 we formally introduce the Restricted Core. We also show some of its properties. Section 5 provides characterizations of the Restricted Core solution. In Section 6 we introduce and

\footnotetext{
${ }^{1}$ Similar, for sequencing situations, Fernández et al. (2005) introduce a drop out monotonic solution that assigns dividends fully to that player of the corresponding coalition who is last in the queue, while the Restricted Core on the ordering from first to last assigns to players earlier in the queue a share in the dividend at least equal to that of later players.
} 
characterize a further refinement of the Restricted Core, the Restricted Selectope which is a solution for games with ordered players that is defined in line with the Selectope and assigns to any game with ordered players a (possibly empty) subset of the Restricted Core. In Section 7 we argue that the Restricted Core provides a reasonable solution for the water distribution problem. The final remarks in Section 8 conclude the paper.

\section{Preliminaries}

\section{$2.1 \quad$ TU-games}

A cooperative game with transferable utility, or simply a TU-game, is a pair $(N, v)$, where $N=\{1, \ldots, n\}$ is a finite set of $n$ players, and $v: 2^{N} \rightarrow \mathbb{R}$ is a characteristic function on $N$ such that $v(\emptyset)=0$. For any coalition $S \subseteq N, v(S)$ is the worth of coalition $S$, i.e., the members of coalition $S$ can obtain a total payoff of $v(S)$ by agreeing to cooperate. Since we take the player set $N$ to be fixed, we represent a TU-game by its characteristic function $v$ and we denote the collection of all TU-games on $N$ by $\mathcal{G}^{N}$. A game $v \in \mathcal{G}^{N}$ is convex if $v(S)+v(T) \leq v(S \cup T)+v(S \cap T)$ for all $S, T \subseteq N$.

A special class of TU-games are the unanimity games. Let $\Omega^{N}=2^{N} \backslash\{\emptyset\}$ denote the collection of all nonempty subsets of $N$. Then, for $T \in \Omega^{N}$, the unanimity game $u_{T}$ on $N$ is given by $u_{T}(S)=1$ if $T \subseteq S$, and $u_{T}(S)=0$ otherwise. Writing $v$ as a $\left(2^{n}-1\right)$-dimensional vector with the worths of the $2^{n}-1$ nonempty coalitions in $\Omega^{N}$ as its components, it is well-known that for every TU-game $v$, there exist unique weights $\Delta_{v}(T) \in \mathbb{R}$ such that $v=\sum_{T \in \Omega^{N}} \Delta_{v}(T) u_{T}$, i.e., $v$ is a unique linear combination of unanimity games. The weights $\Delta_{v}(T), T \in \Omega^{N}$, are usually called the (Harsanyi) dividends of $v$. Recursively, solving $v(S)=\sum_{T \subseteq S} \Delta_{v}(T)$ on the number of players starting from $\Delta_{v}(\{i\})=v(\{i\})$ for the single player coalitions yields $\Delta_{v}(T)=v(T)-\sum_{S \subset T} \Delta_{v}(S)=\sum_{S \subseteq T}(-1)^{|T|-|S|} v(S)$ for $T \subseteq N$ (see Harsanyi (1959)). So, the dividend of a singleton is equal to its worth while, recursively, the dividends of all other coalitions are equal to their worth minus the dividends of all proper subcoalitions. In this sense the dividend of a coalition might be considered as the synergy earnings of cooperation of the coalition that were not yet realized by its proper subcoalitions.

For a collection of games $\mathcal{G} \subseteq \mathcal{G}^{N}$, a set-valued solution $F$ on $\mathcal{G}$ assigns a set $F(v) \subset \mathbb{R}^{n}$ of payoff vectors to every TU-game $v \in \mathcal{G}$. A single-valued solution $f$ on $\mathcal{G}$ assigns precisely one payoff vector $f(v) \in \mathbb{R}^{n}$ to every $v \in \mathcal{G}$. Examples of set-valued solutions are the Core and the Harsanyi set (or Selectope). The Core (Gillies, 1953) of TU-game $v \in \mathcal{G}^{N}$ is the set of all efficient payoff vectors that are stable in the sense that 
no coalition can do better by separating, and is given by

$$
\operatorname{Core}(v)=\left\{x \in \mathbb{R}^{n} \mid \sum_{i \in N} x_{i}=v(N) \text { and } \sum_{i \in S} x_{i} \geq v(S) \text { for all } S \subset N\right\} .
$$

As known, the Core of a game is nonempty if and only if $v$ is balanced, see e.g. Bondareva (1962) or Shapley (1967). Since every convex game is balanced and every totally positive game is convex, it follows that the Core is nonempty on $\mathcal{G}_{+}^{N}$.

The Harsanyi set (Vasil'ev 1978, 1981) of $v \in \mathcal{G}^{N}$ is the set $H(v)$ of payoff vectors obtained by distributing the dividend of any coalition $T \in \Omega^{N}$ in any possible way among the players in $T$. For $T \in \Omega^{N}$, the set of sharing vectors $p^{T} \in \mathbb{R}_{+}^{n}$ is defined as

$$
P^{T}=\left\{\begin{array}{l|l}
p^{T} \in \mathbb{R}^{n} & \begin{array}{l}
\text { (i) } p_{i}^{T}=0 \text { for all } i \in N \backslash T, \\
\text { (ii) } p_{i}^{T} \geq 0 \text { for all } i \in T, \text { and } \\
\text { (iii) } \sum_{i \in T} p_{i}^{T}=1
\end{array}
\end{array}\right\}
$$

and a sharing system is a tuple $p=\left[p^{T}\right]_{T \in \Omega^{N}}$ with $p^{T} \in P^{T}$ for every $T \in \Omega^{N}=2^{N} \backslash\{\emptyset\}$. Then every sharing system $p=\left[p^{T}\right]_{T \in \Omega^{N}}$ yields the corresponding Harsanyi payoff vector of game $v$ given by

$$
\varphi_{i}^{p}(v)=\sum_{T \in \Omega^{N}} \Delta_{v}(T) p_{i}^{T} \text { for all } i \in N,
$$

i.e., the payoff to player $i$ is given by the sum of its shares in the dividends of the game. The Harsanyi set $H(v)$ is the collection of all Harsanyi payoff vectors, thus

$$
H(v)=\left\{\varphi^{p}(v) \in \mathbb{R}^{n} \mid p^{T} \in P^{T} \text { for any } T \in \Omega^{N}\right\} .
$$

It should be noticed that the Harsanyi set of a game is equal to its Selectope, as introduced in Hammer et al. (1977), see also Derks et al. (2000).

A TU-game $v$ is called totally positive if all dividends are nonnegative. (finite totally positive games, as well as their infinite analogs, were introduced in Vasil'ev (1975); for more details see Vasil'ev $(1981,2006)$ ). We denote the collection of all totally positive TU-games on $N$ by $\mathcal{G}_{+}^{N}$. Every totally positive game $v$ is convex. ${ }^{2}$. Moreover, for every $v \in \mathcal{G}^{N}$ it holds that the Harsanyi set is a subset of the Core, and it equals the Core if $v$ is totally positive ${ }^{3}$, for proofs see Vasil'ev (1981), Derks et al. (2000) and Vasil'ev and Van der Laan (2002).

Proposition 2.1 For every $v \in \mathcal{G}^{N}$ it holds that $H(v) \subseteq \operatorname{Core}(v)$. If $v \in \mathcal{G}_{+}^{N}$ then $H(v)=\operatorname{Core}(v)$.

\footnotetext{
${ }^{2}$ In fact, every almost positive game is convex, where a game is almost positive if the dividends of all coalitions with at least two players are nonnegative.

${ }^{3}$ In fact, for every $v \in \mathcal{G}^{N}$ it holds that $H(v)=\operatorname{Core}(v)$ if and only if $v$ is almost totally positive.
} 
Further, for every $v \in \mathcal{G}^{N}, H(v)$ contains the Shapley value of $v$ and thus is nonempty. Also all marginal value vectors of a game $v$ are contained in $H(v)$.

The Shapley value (Shapley (1953)) is the single-valued solution $S h: \mathcal{G}^{N} \rightarrow \mathbb{R}^{N}$ given by

$$
S h_{i}(v)=\sum_{\{S \subseteq N \mid i \in S\}} \frac{\Delta_{v}(S)}{|S|} \text { for every } i \in N .
$$

So, for every game $v$ the Shapley value yields the Harsanyi payoff vector that is obtained by taking the sharing vectors $p^{T}$ given by $p_{i}^{T}=\frac{1}{|T|}$ for all $i \in T$ and every $T \in \Omega^{N}$, and thus $\operatorname{Sh}(v)$ is in $H(v)$.

Alternatively, the Shapley value is defined as the average of all marginal value vectors over all permutations of the players. For a permutation $\pi: N \rightarrow N$, assigning rank number $\pi(i) \in N$ to any player $i \in N$, we define $\pi^{i}=\{j \in N \mid \pi(j) \leq \pi(i)\}$, i.e., $\pi^{i}$ is the set of all players with rank number at most equal to the rank number of $i$, including $i$ itself. Then the marginal value vector $m^{\pi}(v) \in \mathbb{R}^{n}$ of game $v$ and permutation $\pi$ is given by $m_{i}^{\pi}(v)=v\left(\pi^{i}\right)-v\left(\pi^{i} \backslash\{i\}\right), i \in N$. For convex games each marginal value vector lies in the Core of the game. For every game each marginal vector lies in the Harsanyi set. More precisely, for permutation $\pi$ and coalition $T \in \Omega^{N}$, let $i(T)$ be the player in $T$ such that $\pi(j) \leq \pi(i(T))$ for all $j \in T$, i.e., $i(T)$ is the player in $T$ with the highest rank number. Then $m^{\pi}(v) \in H(v)$ is the Harsanyi payoff vector corresponding to the sharing system $p=\left[p^{T}\right]_{T \in \Omega^{N}}$ with $p^{T}(\pi) \in P^{T}$ defined by $p_{i(T)}^{T}(\pi)=1$ and $p_{j}^{T}(\pi)=0$ for all $j \neq i(T)$ (see e.g. Derks et al. 2006).

\subsection{Games with ordered players}

In this paper we assume the players to be part of a hierarchical structure that is represented by a directed graph. A directed graph or digraph is a pair $(N, D)$ where $N=\{1, \ldots, n\}$ is a finite set of nodes (representing the players) and $D \subseteq N \times N$ is a binary relation on $N$. We assume $D$ to be irreflexive, i.e., $(i, i) \notin D$ for all $i \in N$. The collection of all (irreflexive) binary relations on $N$ is denoted by $\mathcal{D}^{N}$. Observe that $D$ is a collection of ordered pairs. Since we take the finite set $N$ to be fixed, we represent a digraph on $N$ by its collection $D$ of ordered pairs. For $i, j \in N$, a path between $i$ and $j$ in $D$ is a sequence of different nodes $\left(i_{1}, \ldots, i_{m}\right)$ such that $i_{1}=i, i_{m}=j$, and $\left\{\left(i_{k}, i_{k+1}\right),\left(i_{k+1}, i_{k}\right)\right\} \cap D \neq \emptyset$ for $k=1, \ldots, m-1$. A path $\left(i_{1}, \ldots, i_{m}\right)$ is a directed path if $\left(i_{k}, i_{k+1}\right) \in D$ for $k=1, \ldots, m-1$. A directed path $\left(i_{1}, \ldots, i_{m}\right)$ is a cycle in $D$ if $m \geq 2$ and $\left(i_{m}, i_{1}\right) \in D$. We call digraph $D$ acyclic if there are no cycles.

A game with ordered players is a pair $(v, D)$ with $v \in \mathcal{G}^{N}$ a characteristic function and $D \in \mathcal{D}^{N}$ a directed graph with set $N$ as its set of nodes. In this paper we only consider 
pairs $(v, D)$ with $v \in \mathcal{G}_{+}^{N}$ a totally positive game, however we often just shortly refer to a pair $(v, D) \in \mathcal{G}_{+}^{N} \times \mathcal{D}^{N}$ as a game with ordered players. The digraph $D$ represents an ordered structure on the set of players. Player $i \in N$ dominates player $j \in N$ if $(i, j) \in D$, i.e., when, in the ordering $i$ is a predecessor of $j$ (and thus $j$ a successor of $i$ ). Observe that we allow that $D$ contains cycles, so that it is possible that a player $i_{1}$ has a successor $i_{2}, i_{2}$ has a successor $i_{3}$ and so on to some player $i_{m}$ that is a predecessor of $i_{1}$. If $m=2$, then $i_{1}$ is a predecessor of $i_{2}$ and $i_{2}$ of $i_{1}$. Although one might argue that an order should satisfy certain properties, for instance the ordering is a hierarchical structure without cycles, in this paper we refer to any structure that is represented by a digraph as an order, including the empty digraph $D=\emptyset$ and the complete digraph $D=\{(i, j) \mid i, j \in N, i \neq j\}$. We already remark here that the characterization results in Sections 5 and 6 will hold for any subclass $\mathcal{E} \subseteq \mathcal{D}^{N}$ of digraphs, so also if one restricts to acyclic structures, rooted trees, and even to classes of digraphs that consist of only one digraph.

For a collection $\mathcal{G} \times \mathcal{E} \subseteq \mathcal{G}^{N} \times \mathcal{D}^{N}$ of games with ordered players, a solution $\mathcal{F}: \mathcal{G} \times$ $\mathcal{E} \rightarrow \mathbb{R}^{n}$ assigns a set $\mathcal{F}(v, D) \subset \mathbb{R}^{n}$ of payoff vectors to every $(v, D) \in \mathcal{G} \times \mathcal{E}$. Possible solutions that do not take into account the ordering of the players are for instance $\mathcal{F}(v, D)=$ $\operatorname{Core}(v)$ or $\mathcal{F}(v, D)=H(v)$ for every $(v, D) \in \mathcal{G} \times \mathcal{E}$. For the class of games $\mathcal{G}_{+}^{N} \times \mathcal{D}^{N}$ of totally positive games with ordered players, in Section 4 we introduce a solution that distributes the Harsanyi dividends in such a way that higher ordered players get at least the same share in a dividend than lower ranked players in the corresponding coalition. In the next section we first discuss a motivating example of a totally positive game with ordered players.

We conclude this subsection with a few further notions on directed graphs. For $T \in \Omega^{N}$, the subgraph of $(N, D)$ restricted to $T$ is given by $(T, D(T))$ with $D(T)=$ $\{(i, j) \in D \mid i, j \in T\}$. A set of nodes $T \subseteq N$ is connected in $D$ if, for every $i, j \in T$ there is a path $\left(i_{1}, \ldots, i_{m}\right)$ between $i$ and $j$ such that $\left\{i_{1}, \ldots, i_{m}\right\} \subseteq T$, i.e., between every two nodes in $T$ there is a path in $D$ that only contains nodes of $T$. A set $S \in \Omega^{N}$ is comprehensive from above in $D$ if $[j \in S$ and $(i, j) \in D]$ implies that $i \in S$, i.e., for every player $j$ in $S$ also all predecessors of $j$ are in $S$. A set $S$ is complete in $D$ when $S$ is connected and comprehensive from above in $D$. Finally, for nonempty $T \subseteq N$, we denote $\mathcal{C}_{D}^{T}$ as the set of complete subsets of $T$ in the subgraph $(T, D(T))$.

\section{The water distribution problem}

In their paper 'Sharing a river', Ambec and Sprumont (2002) consider the problem of the optimal distribution of water to agents located along a river from upstream to downstream. Let $N=\{1, \ldots, n\}$ be the set of players representing the agents on the river, numbered 
successively from upstream to downstream, and let $e_{i} \geq 0$ be the flow of water entering the river between player $i-1$ and $i, i=1, \ldots, n$, with $e_{1}$ the inflow before the most upstream player 1. Further it is assumed that each player has a quasi-linear utility function given by $u^{i}\left(x_{i}, t_{i}\right)=b^{i}\left(x_{i}\right)+t_{i}$ where $t_{i}$ is a monetary compensation to player $i, x_{i}$ is the amount of water allocated to player $i$, and $b^{i}: \mathbb{R}_{+} \rightarrow \mathbb{R}$ is a continuous nondecreasing function yielding the benefit $b^{i}\left(x_{i}\right)$ to player $i$ of the consumption $x_{i}$ of water. An allocation is a pair $(x, t) \in \mathbb{R}_{+}^{n} \times \mathbb{R}^{n}$ of water distribution and compensation scheme, satisfying

$$
\sum_{i=1}^{n} t_{i} \leq 0 \quad \text { and } \quad \sum_{i=1}^{j} x_{i} \leq \sum_{i=1}^{j} e_{i}, j=1, \ldots, n .
$$

The first condition is a budget condition and says that the total amount of compensations is nonpositive, i.e., the compensations only redistribute the total welfare. The second condition reflects that any player can use the water that entered upstream, but that the water inflow downstream of some player can not be allocated to this player. So, for any $j$, the sum of the water uses $x_{1}, \ldots, x_{j}$ is at most equal to the sum of the inflows $e_{1}, \ldots, e_{j}$.

Because of the quasi-linearity and the possibility of making money transfers, an allocation is Pareto optimal (efficient) if and only if the distribution of the water streams maximizes the total benefits, i.e., the optimal water distribution $x^{*} \in \mathbb{R}_{+}^{n}$ solves the maximization problem:

$$
\max _{x_{1}, \ldots, x_{n}} \sum_{i=1}^{n} b^{i}\left(x_{i}\right) \text { s.t. } \sum_{i=1}^{j} x_{i} \leq \sum_{i=1}^{j} e_{i}, \quad j=1, \ldots, n, \text { and } x_{i} \geq 0, i=1, \ldots, n .
$$

A welfare distribution allocates the total benefits of an optimal water distribution $x^{*}$ over the players, i.e., it is a vector $z \in \mathbb{R}^{n}$ assigning utility $z_{i}$ to player $i$ and satisfying $\sum_{i=1}^{n} z_{i}=$ $\sum_{i=1}^{n} b^{i}\left(x_{i}^{*}\right)$. Clearly, any welfare distribution $z$ can be implemented by the allocation $(x, t)$ with $x_{i}=x_{i}^{*}$ and $t_{i}=z_{i}-b^{i}\left(x_{i}^{*}\right), i=1, \ldots, n$.

The problem to find a 'fair' welfare distribution can be modelled by the following game $(N, v)$. Obviously, the worth $v(N)$ is given by $v(N)=\sum_{i=1}^{n} b^{i}\left(x_{i}^{*}\right)$ with $x^{*} \in \mathbb{R}_{+}^{n}$ a solution of the maximization problem (3.1). Further, for any pair of players $i, j$ with $j>i$ it holds that water inflow entering the river before the upstream player $i$ can only be allocated to the downstream player $j$ if all players between $i$ and $j$ cooperate, otherwise any player between $i$ and $j$ can take the flow from $i$ to $j$ for its own use. Hence, a coalition $T$ is admissible if and only if $T$ is a coalition of consecutive players along the river, i.e., $T=\{i, i+1, \ldots, j\}$. In the sequel we denote such a coalition of consecutive players by $[i, j]$. For any consecutive coalition $[i, j]$ its worth $v([i, j])$ is given by

$$
v([i, j])=\sum_{h=i}^{j} b^{h}\left(x_{h}^{[i, j]}\right) \text { where } x^{[i, j]}=\left(x_{h}^{[i, j]}\right)_{h=i}^{j} \text { solves }
$$




$$
\max _{x_{i}, \ldots, x_{j}} \sum_{h=i}^{j} b^{h}\left(x_{h}\right) \text { s.t. } \sum_{k=i}^{h} x_{k} \leq \sum_{k=i}^{h} e_{k}, \quad h=i, \ldots, j, \text { and } x_{k} \geq 0, k=i, \ldots, j .
$$

For any other (non-consecutive) coalition $S$ it holds that $v(S)$ is equal to the sum of the worths of its maximal consecutive subsets ${ }^{4}$. We refer to this game as the river game. In the sequel we take the following assumption from Ambec and Sprumont (2002).

Assumption 3.1 In the river game every benefit function $b^{i}: \mathbb{R}_{+} \rightarrow \mathbb{R}$ is differentiable with derivative going to infinity as $x_{i}$ tends to zero, strictly increasing and strictly concave.

Under this assumption the river game is a totally positive game. This follows from the following more general result. We say that a TU-game $v \in \mathcal{G}^{N}$ is a line-graph game on linear order $(1,2, \ldots, n)$ if for every coalition $S \subseteq N$ it holds that $v(S)$ equals the sum of the worths of all maximal consecutive subsets of $S$, see Greenberg and Weber (1986). Note that this is equivalent to saying that the dividend of every nonconsecutive coalition is zero, see e.g. Owen (1986) and Bilbao (1998). It turns out that for this class of games, convexity is equivalent to totally positiveness.

Proposition 3.2 Let $v$ be a line-graph game with $v(\{i\}) \geq 0$ for all $i \in N$. Then $v$ is convex if and only if $v$ is totally positive.

Proof. It is known that a totally positive TU-game is convex. So, we only have to show that for line-graph games also the reverse holds. Therefore, let $v$ be a convex line-graph game with $v(\{i\}) \geq 0$ for all $i \in N$. From $v(\{i\}) \geq 0$ it follows that $\Delta_{v}(\{i\}) \geq 0, i \in N$. In van den Brink et al. (2007) it is shown that the dividend of a consecutive coalition $S=[i, j]$ consisting of at least two players, is given by

$$
\Delta_{v}([i, j])=v([i, j])-v([i+1, j])-v([i, j-1])+v([i+1, j-1]) .
$$

With $S=[i, j]$ and $T=[i+1, j-1]$ (with $T=\emptyset$ if $j<i+2)$, the convexity of $v$ implies that $v([i, j])+v([i+1, j-1]) \geq v([i+1, j])+v([i, j-1])$. Hence it follows that that $\Delta_{v}([i, j]) \geq 0$ for any consecutive coalition $[i, j], i \neq j$. As stated already above, any nonconsecutive coalition has zero dividend. So, $v$ is totally positive.

Although an arbitrary convex game $v$ does not need to be totally positive, Proposition 3.2 shows that for line-graph games these properties are equivalent. Under Assumption 3.1, Ambec and Sprumont (2002) have shown that the river game is convex. Clearly, the river game is also a line-graph game on the linear order $(1,2, \ldots, n)$ and thus Proposition 3.2 applies. Further recall from Proposition 2.1 that $H(v)=\operatorname{Core}(v)$ when $v$ is totally positive. So we have the following corollary.

\footnotetext{
${ }^{4}$ A subset $T$ of $S$ is maximal consecutive if $T$ is consecutive and $T \cup h$ is not consecutive for any $h \in S \backslash T$.
} 
Corollary 3.3 Let $v$ be the river game associated to a water distribution problem with benefit functions satisfying Assumption 3.1. Then $v$ is totally positive, $\Delta_{v}(T)=0$ if $T$ is nonconsecutive, and $H(v)=\operatorname{Core}(v)$.

Ambec and Sprumont (2002) propose as solution for the water distribution problem the vector $m^{u}(v)$, being the marginal value vector $m^{\pi}$ with respect to the permutation $\pi(i)=i$ for all $i \in N$, i.e., the vector given by $m_{1}^{u}(v)=v(\{1\})$ and $m_{i}^{u}(v)=v([1, i])-$ $v([1, i-1]), i=2, \ldots, n$. They show that this is the unique payoff vector that is both core-stable (i.e., lies in the Core) and fair. This fairness property means that no coalition $S$ gets a payoff above its aspiration level, defined as the maximum worth $S$ can attain by an optimal distribution among its members of the water inflows of all players $1, \ldots, s$, where $s=\max \{h \mid h \in S\}$, so also using the water inflows of the players not in $S$, but upstream to the most downstream member of $S$. So, the fairness property requires that the total payoff to coalition $[i, j]$ is at most $v([i, j])$. On the other hand, core-stableness requires that coalition $[1, j]$ receives at least $v([1, j])$. Therefore, core-stableness and fairness imply that the total payoff to the players in coalition $[1, j]$ should be equal to $v([1, j])$, which yields $m^{u}(v)$ as the unique outcome. Observing that $m^{u}(v)$ is the marginal value vector of the game with respect to the permutation $\pi(i)=i$ for all $i$, it indeed follows from the convexity of the game that $m^{u}(v)$ is in the Core of the game.

Although core-stableness and fairness seem to be reasonble properties, the unique outcome resulting from these two properties is quite counterintuitive. For every $i<n$, we have that the total payoff of the player in the consecutive coalition $[i, j]$ upstream of $i$ (including $i$ itself) is equal to $v([1, i])$, while the total payoff to the downstream coalition $[i+1, n]$ is equal to $v(N)-v([1, i]) \geq v([i+1, n])$, i.e., all additional profit that is realised when the two coalitions $[1, i]$ and $[i+1, n]$ merge to the grand coalition $N$ goes to the downstream coalition. However, any upstream coalition $[1, i]$ can prevent that coalition $[i+1, n]$ gets more than $v[i+1, n]$ by using all inflows $e_{1}, \ldots, e_{i}$ by itself. So, although the coalition $[1, i]$ can play some type of ultimatum game by claiming that they will use their total water inflow $\sum_{h=1}^{i} e_{i}$ by themselves unless the players of the downstream coalition $[i+1, j]$ are willing to give almost all profit of cooperation to the upstream coalition, the solution proposed by Ambec and Sprumont does the reverse, all profit goes to the downstream coalition. Putting it differently, recall from the end of Subsection 2.1 that the marginal value vector $m^{u}(v)$ corresponding to permutation $\pi(i)=i$ is also obtained by assigning the nonnegative dividend $\Delta_{v}([i, j])$ of any consecutive coalition $[i, j]$ to the most downstream player $j$ in that coalition. It shows that the Ambec and Sprumont solution has a drawback that a player is not rewarded for letting pass the water from its upstream players to its downstream players and thus does not give any incentive to a player $i$ to cooperate with its successors downstream along the river. Even if one agrees with the 
idea that downstream players should get a share in the dividends at least as high as the shares of upstream players, it seems that giving dividends fully to downstream players is too extreme.

Alternatively, the vector $m^{l}(v)$ being the marginal value vector $m^{\pi}$ with respect to the permutation $\pi(i)=n-i+1$ assigns every dividend $\Delta_{v}([i, j])$ fully to the most upstream player $i$ and yields payoffs $m_{n}^{l}(v)=v(\{n\})$ and $m_{i}^{l}(v)=v([i, n])-v([i+1, n])$ for all $i=1, \ldots, n-1$. Maybe assigning every dividend to the most upstream player in the coalition is also too extreme (of course, not more extreme than assigning it to the most downstream player), but since any upstream coalition $[1, i]$ can prevent that any downstream coalition $[i+1, n]$ gets more than $v([i+1, n])$ by distributing all its water inflows among themselves, it seems to be reasonable that for two players $h$ and $k$ in a consecutive coalition $T$, the share of player $h$ in the dividend of coalition $T$ is at least equal to the share of $k$ when player $k$ is located downstream to player $h$.

In the next sections we will see that the latter outcomes are indeed obtained by applying the Restricted Core for games with nonnegative dividends (which is the solution introduced in this paper) to the river game. Therefore, we consider the linear ordering $D$ given by

$$
D=\{(i, j) \in N \times N \mid j=i+1\},
$$

in which the players along the river are ordered from upstream to downstream, and consider the game with ordered players $(v, D)$, where $v$ is the totally positive river game given by (3.1) and (3.2). So $D$ represents the situation that any player $j$ needs the cooperation of its upstream neighbor $i=j-1$ to receive water from its upstream players $1, \ldots, j-1$. Observe that a coalition $S$ is connected in $D$ if and only if $S$ is a coalition $[i, j]$ of consecutive players. Therefore, only connected coalitions in $D$ have a nonzero dividend in the river game. The Restricted Core consists of all payoff vectors obtained by requiring that, for every consecutive coalition $[i, j]$, the share of player $h \in[i, j-1]$ in the dividend of coalition $[i, j]$ is at least equal to the share of it downstream neighbour $h+1$. Since for totally positive games every allocation obtained by distributing the dividends belongs to the Core of the game, by restricting the way in which the dividends are distributed we obtain a refinement of the Core.

\section{The Restricted Core}

In this section we propose a solution $\mathcal{F}$ on the class $\mathcal{G}_{+}^{N} \times \mathcal{D}^{N}$ of totally positive games with ordered players. As suggested in the previous section, the solution distributes dividends among players in such a way that higher ordered players get at least the same share in a 
dividend than lower ranked players in the corresponding coalition. So, considering a totally positive TU-game $v \in \mathcal{G}_{+}^{N}$ and a digraph $D \in \mathcal{D}^{N}$, we require for every coalition $T$ and two players $i, j \in T$ that player $i$ gets at least as much as player $j$ from the dividend $\Delta_{v}(T)$ if $(i, j) \in D$. This is obtained by restricting ourselves to sharing systems $p=\left[p^{T}\right]_{T \in \Omega^{N}}$ such that for any $T \in \Omega^{N}$ the sharing vector $p^{T}$ belongs to the subset $P_{D}^{T}$ of $P^{T}$ given by

$$
P_{D}^{T}=\left\{p^{T} \in P^{T} \mid p_{i}^{T} \geq p_{j}^{T} \text { for all } i, j \in T \text { with }(i, j) \in D\right\} .
$$

Now we propose as solution concept for totally positive games with ordered players the set of all Harsanyi payoff vectors corresponding to a sharing system $p=\left[p^{T}\right]_{T \in \Omega^{N}}$ with $p^{T} \in P_{D}^{T}$ for all $T \in \Omega^{N}$. Since for totally positive games the Harsanyi set is equal to the Core, the collection of all these Harsanyi payoff vectors is a subset of the Core. Therefore the solution is called the Restricted Core and denoted by RC.

Definition 4.1 The Restricted Core of a totally positive game with ordered players $(v, D) \in$ $\mathcal{G}_{+}^{N} \times \mathcal{D}^{N}$ is the set

$$
R C(v, D)=\left\{x \in \mathbb{R}^{n} \mid x=\sum_{T \in \Omega^{N}} \Delta_{v}(T) p^{T}, p^{T} \in P_{D}^{T} \text { for any } T \in \Omega^{N}\right\} .
$$

Example 4.2 Consider a market situation with two buyers and one seller of a good. The buyers both want only one item of the good, and the seller ownd two items of the good. Both buyers are prepared to pay 1 for an item, while the reservation value of the seller is zero. This situation can be represented by the TU-game $v$ on $N=$ $\{1,2,3\}$ with player 1 being the seller and the other two players being the buyers, with $v$ given by $v(N)=2, v(\{1, j\})=1$ if $j \in\{2,3\}$, and $v(S)=0$ otherwise. Since $v=u_{\{1,2\}}+u_{\{1,3\}}$, the coalitions $\{1,2\}$ and $\{1,3\}$ have dividend 1 , while the dividends of all other coalitions is zero. The unrestricted Core of this game is given by ${ }^{5} \operatorname{Core}(v)=$ $\operatorname{Conv}\left(\left\{(2,0,0)^{\top},(1,0,1)^{\top},(1,1,0)^{\top},(0,1,1)^{\top}\right\}\right)$, see Figure 1.

Now, consider the game with ordered players $(v, D)$ on $N=\{1,2,3\}$ with $v$ as given before and $D=\{(1,3),(2,3)\}$. The Restricted Core is given by $\operatorname{RC}(v, D)=\left\{x=\left(x_{1}, x_{2}, x_{3}\right)^{\top} \in\right.$ $\mathbb{R}^{3} \mid x_{1}=p_{1}^{\{1,2\}}+p_{1}^{\{1,3\}}, x_{2}=p_{2}^{\{1,2\}}, x_{3}=p_{3}^{\{1,3\}}$ and $\left.p^{T} \in P_{D}^{T}, T \in \Omega^{N}\right\}$ with $P_{D}^{\{1,3\}}=$ $\left\{p^{\{1,3\}} \in P^{\{1,3\}} \mid p_{1}^{\{1,3\}} \geq p_{3}^{\{1,3\}}\right\}$ and $P_{D}^{\{2,3\}}=\left\{p^{\{2,3\}} \in P^{\{2,3\}} \mid p_{2}^{\{2,3\}} \geq p_{3}^{\{2,3\}}\right\}$. It follows that $\mathrm{RC}(v, D)=\operatorname{Conv}\left(\left\{(2,0,0)^{\top},(1,1,0)^{\top},\left(\frac{1}{2}, 1, \frac{1}{2}\right)^{\top},\left(1 \frac{1}{2}, 0, \frac{1}{2}\right)^{\top}\right\}\right)$. This is the dashed subset of $\operatorname{Core}(v)$, see Figure 1. Note that the Shapley value $\operatorname{Sh}(v)=\left(1, \frac{1}{2}, \frac{1}{2}\right)^{\top}$ of game $v$ belongs to $R C(v, D)$.

\footnotetext{
${ }^{5}$ In the sequel, $\operatorname{Conv}(A)$ denotes the convex hull of $A \subset \mathbb{R}^{n}$.
} 


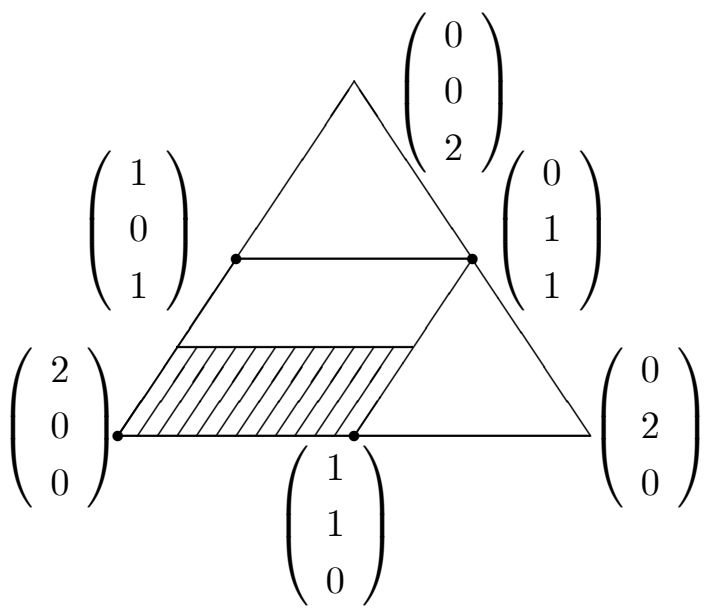

Figure 1: $\operatorname{Core}(v)$ and $\mathrm{RC}(v, D)$ of Example 4.2 .

In Example 4.2 the Restricted Core of the game with ordered players appears to be a subset of the Core of the original game. Moreover, the Shapley value of the unrestricted game belongs to the Restricted Core. Both these facts hold in general. ${ }^{6}$

Theorem 4.3 For every $(v, D) \in \mathcal{G}_{+}^{N} \times \mathcal{D}^{N}$ it holds that $\operatorname{Sh}(v) \in R C(v, D) \subseteq \operatorname{Core}(v)$.

Proof. Let $(v, D) \in \mathcal{G}_{+}^{N} \times \mathcal{D}^{N}$. Take $p_{i}^{T}=\frac{1}{|T|}$ if $i \in T$, and $p_{i}^{T}=0$ otherwise. Clearly, $\sum_{T \in \Omega^{N}} \Delta_{v}(T) p^{T}=\operatorname{Sh}(v)$ and $p^{T} \in P_{D}^{T}$ for every $D \in \mathcal{D}^{N}$ and $T \in \Omega^{N}$. Thus, $\operatorname{Sh}(v) \in$ $\mathrm{RC}(v, D)$. The inclusion $\mathrm{RC}(v, D) \subseteq$ Core $(v)$ follows immediately from $P_{D}^{T} \subseteq P^{T}, T \in \Omega^{N}$.

Note that $\operatorname{Sh}(v) \in \mathrm{RC}(v, D)$ implies that $\mathrm{RC}(v, D) \neq \emptyset$ for all $(v, D) \in \mathcal{G}_{+}^{N} \times$ $\mathcal{D}^{N}$. The Restricted Core $\operatorname{RC}(v, D)$ generalizes the Shapley value and the Core for totally positive TU-games in the sense that the corresponding inclusions in Theorem 4.3 can be equalities. Let $D^{c}$ denote the complete irreflexive digraph given by $D^{c}=\{(i, j) \in N \times N \mid$ $i \neq j\}$.

Theorem 4.4 Let $D \in \mathcal{D}^{N}$. Then

(i) $R C(v, D)=\{\operatorname{Sh}(v)\}$ for every $v \in \mathcal{G}_{+}^{N}$ if and only if $D=D^{c}$;

(ii) $R C(v, D)=$ Core $(v)$ for every $v \in \mathcal{G}_{+}^{N}$ if and only if $D=\emptyset$.

Proof. (i) If When $D=D^{c}$, then $(i, j) \in D$ for all $i, j, i \neq j$. Thus for all $T \in \Omega^{N}$, we have $P_{D}^{T}=\left\{p^{T} \in P^{T} \mid p_{i}^{T}=p_{j}^{T}\right.$ for all $\left.i, j \in T\right\}=\left\{p^{T} \in P^{T} \mid p_{i}^{T}=\frac{1}{|T|}\right.$ for all $\left.i \in T\right\}$. So $\operatorname{RC}(v, D)=\{S h(v)\}$. Only if Suppose that $D \neq D^{c}$, i.e., there exist $i, j \in N, i \neq j$,

\footnotetext{
${ }^{6}$ We remark that Theorems 4.3 and 4.4 hold for any game (not necessarily totally positive) with ordered players, but then with respect to the Restricted Harsanyi Set as introduced in van den Brink et al. (2006).
} 
with $(i, j) \notin D$. Take $v=u_{\{i, j\}}$ and let $e^{j} \in \mathbb{R}^{N}$ be given by $e_{j}^{j}=1$ and $e_{h}^{j}=0$ for all $h \in N \backslash\{j\}$. Then $e^{j} \in \operatorname{RC}(v, D)$, but $e^{j} \neq S h(v)$.

(ii) If Take $D=\emptyset$. Then $P_{D}^{T}=P^{T}$ for every $T \in \Omega^{N}$, implying that $\operatorname{RC}(v, D)=H(v)=$ $\operatorname{Core}(v)$, where the latter equality follows from $v \in \mathcal{G}_{+}^{N}$. Only if Suppose that $D \neq \emptyset$, i.e., there exist $i, j \in N, i \neq j$, with $(i, j) \in D$. Again, take $v=u_{\{i, j\}}$ and consider $e^{j} \in \mathbb{R}^{N}$. Then $e^{j} \in \operatorname{Core}(v)$ although $e^{j} \notin \mathrm{RC}(v, D)$.

For every $(v, D) \in \mathcal{G}_{+}^{N} \times \mathcal{D}^{N}$ the set of payoff vectors $\mathrm{RC}(v, D)$ is a polytope in $\mathbb{R}^{n}$. To find the extreme points of $\operatorname{RC}(v, D)$, the following theorem from van den Brink et al. (2008) is useful and will be applied later. For $S \subseteq N$, let $a^{S} \in \mathbb{R}^{n}$ be the vector with components $a_{i}^{S}=\frac{1}{|S|}$ when $i \in S$ and $a_{i}^{S}=0$, otherwise. The theorem characterizes the extreme points of $P_{D}^{T}$ as those vectors $a^{S}$ corresponding to complete subsets of $T$.

Theorem 4.5 (van den Brink et al. 2008)

For $T \subseteq N$, the set of extreme points of $P_{D}^{T}$ is given by

$$
\operatorname{Ex}\left(P_{D}^{T}\right)=\left\{a^{S} \in \mathbb{R}^{n} \mid S \in \mathcal{C}_{D}^{T}\right\} .
$$

\section{Characterization of the Restricted Core}

We first state four axioms for a solution $\mathcal{F}$ on $\mathcal{G}_{+}^{N} \times \mathcal{D}^{N}$, that are generalizations of standard axioms in cooperative game theory as discussed for standard TU-games in Vasil'ev (1981) and Vasil'ev and van der Laan (2002).

Axiom (Efficiency) For all $(v, D) \in \mathcal{G}_{+}^{N} \times \mathcal{D}^{N}$, any payoff vector in the solution is efficient, i.e., $\sum_{i \in N} x_{i}=v(N)$ for all $x \in \mathcal{F}(v, D)$.

Recall that a player $i \in N$ is a null player in $v \in \mathcal{G}^{N}$ if $v(S)=v(S \backslash\{i\})$ for all $S \subseteq N$.

Axiom (Null player property) For all $(v, D) \in \mathcal{G}_{+}^{N} \times \mathcal{D}^{N}$, any payoff vector $x \in \mathcal{F}(v, D)$ satisfies $x_{i}=0$, whenever $i$ is a null player in $v$.

Axiom (Additivity) For all $v, w \in \mathcal{G}_{+}^{N}$ and $D \in \mathcal{D}^{N}$, it holds that $\mathcal{F}(v+w, D)=$ $\mathcal{F}(v, D)+\mathcal{F}(w, D)$, where $(v+w)(S)=v(S)+w(S)$ for all $S \subseteq N$.

Axiom (Nonnegativity) For all $(v, D) \in \mathcal{G}_{+}^{N} \times \mathcal{D}^{N}$, it holds that $\mathcal{F}(v, D) \subseteq \mathbb{R}_{+}^{n}$.

The next axiom reflects the ordering of the players within the digraph. Since the players payoffs should depend on their contributions in the game $v$ as well as their positions in 
the digraph $D$, we cannot say that players should always earn at least as much as their successors. However, if a player vetoes one of its successors, then we require that this vetoing predecessor earns at least as much as its vetoed successor ${ }^{7}$. Player $i$ vetoes player $j \in N \backslash\{i\}$ in game $v$ if $v(S)=v(S \backslash\{j\})$ for all $S \subseteq N \backslash\{i\}$, i.e., $i$ vetoes $j$ if the marginal contribution of $j$ to any coalition not containing $i$ is equal to zero.

Axiom (Structural monotonicity) For all $(v, D) \in \mathcal{G}_{+}^{N} \times \mathcal{D}^{N}$, any payoff vector $x \in$ $\mathcal{F}(v, D)$ satisfies $x_{i} \geq x_{j}$ whenever $(i, j) \in D$ and $i$ vetoes $j$ in $v$.

The next lemma says that each coalition containing $j$ but not $i$ has zero dividend when $i$ vetoes $j$.

Lemma 5.1 If $i$ vetoes $j$ in $v \in \mathcal{G}_{+}^{N}$, then $\Delta_{v}(T)=0$ for any $T \subseteq N \backslash\{i\}$ such that $j \in T$.

Proof. If $i$ vetoes $j$ in $v \in \mathcal{G}_{+}^{N}$ then $\Delta_{v}(\{j\})=v(\{j\})=0$. Now, consider $T \subseteq N \backslash\{i\}$ with $j \in T$. For $T=\{j\}, \Delta_{v}(T)=v(T)=0$. Proceeding by induction, assume that $\Delta_{v}\left(T^{\prime}\right)=0$ for all $T^{\prime} \subset T$ with $j \in T^{\prime}$. Then $\Delta_{v}(T)=v(T)-\sum_{\substack{S \subset T \\ S \neq \emptyset}} \Delta_{v}(S)=v(T)-$ $\sum_{\substack{S \subseteq T \backslash\{j\} \\ S \neq \emptyset}} \Delta_{v}(S)-\sum_{\substack{S \subset T \\ j \in S}} \Delta_{v}(S)=v(T)-v(T \backslash\{j\})-0$ by the induction hypothesis. But then $\Delta_{v}(T)=0$ since $v(T)=v(T \backslash\{j\})$ by $i$ vetoing $j$ and $T \subseteq N \backslash\{i\}$.

The next theorem says that the Restricted Core solution satisfies the above five axioms on the class $\mathcal{G}_{+}^{N} \times \mathcal{D}^{N}$ of totally positive games with ordered players.

Theorem 5.2 The Restricted Core solution RC satisfies efficiency, the null player property, additivity, nonnegativity and structural monotonicity on $\mathcal{G}_{+}^{N} \times \mathcal{D}^{N}$.

Proof. RC satisfying efficiency, the null player property, additivity and nonnegativity follows similar as this is shown for corresponding properties of the Harsanyi set solution $H$ for TU-games on $\mathcal{G}^{N}$ in Vasil'ev and van der Laan (2002). To show structural monotonicity, for $(v, D) \in \mathcal{G}_{+}^{N} \times \mathcal{D}^{N}$, let $v=\sum_{T \in \Omega^{N}} \Delta_{v}(T) u_{T}$, and let $i, j \in N$ be such that $i$ vetoes $j$ in $v$ and $(i, j) \in D$. For some $T$, let $x^{T}$ be an element in $\operatorname{RC}\left(\Delta_{v}(T) u_{T}, D\right)$. Then there is a $p^{T} \in P_{D}^{T}$ such that $x_{h}^{T}=\Delta_{v}(T) p_{h}^{T}$ for all $h \in T$. We consider four cases.

(i) If $i, j \in T$, then $x_{i}^{T}=\Delta_{v}(T) p_{i}^{T} \geq \Delta_{v}(T) p_{j}^{T}=x_{j}^{T}$ (with equality if also $(j, i) \in D$ ) since $p_{i}^{T} \geq p_{j}^{T}$ and $\Delta_{v}(T) \geq 0$.

(ii) If $i \in T, j \notin T$, then $x_{i}^{T} \geq 0=x_{j}^{T}$ since $\Delta_{v}(T) \geq 0$.

(iii) If $i \notin T, j \in T$, then $\Delta_{v}(T)=0$ by Lemma 5.1 and thus $x_{j}^{T}=0=x_{i}^{T}$.

(iv) If $i, j \notin T$, then $x_{i}^{T}=x_{j}^{T}=0$.

\footnotetext{
${ }^{7}$ For point-valued solutions this is weaker than a similar property introduced in van den Brink and Gilles (1996) who do not require the predecessor to veto the successor.
} 
So, in all cases we have that $x_{i}^{T} \geq x_{j}^{T}$ when $x^{T} \in \mathrm{RC}\left(\Delta_{v}(T) u_{T}, D\right), i$ vetoes $j$ in $v$ and $(i, j) \in D$. By RC satisfying additivity we have that $\mathrm{RC}(v, D)=\sum_{T \in \Omega^{N}} \mathrm{RC}\left(\Delta_{v}(T) u_{T}, D\right)$ for every $(v, D) \in \mathcal{G}_{+}^{N} \times \mathcal{D}^{N}$. So, for each $x \in \operatorname{RC}(v, D)$, there exist $x^{T} \in \operatorname{RC}\left(\Delta_{v}(T) u_{T}, D\right)$, $T \in \Omega^{N}$, such that $x=\sum_{T \in \Omega^{N}} x^{T}$. Since $x_{i}^{T} \geq x_{j}^{T}$ for all $T$, structural monotonicity is satisfied.

Next we show that on the class of totally positive games with ordered players the Restricted Core solution is the maximal (with respect to set inclusion) solution that satisfies the five axioms mentioned above.

Theorem 5.3 If solution $\mathcal{F}$ on $\mathcal{G}_{+}^{N} \times \mathcal{D}^{N}$ satisfies efficiency, the null player property, additivity, nonnegativity and structural monotonicity, then $\mathcal{F}(v, D) \subseteq R C(v, D)$ for all $(v, D) \in \mathcal{G}_{+}^{N} \times \mathcal{D}^{N}$.

Proof. Suppose that solution $\mathcal{F}$ on $\mathcal{G}_{+}^{N} \times \mathcal{D}^{N}$ satisfies the five properties. For $x \in$ $\mathcal{F}\left(\Delta_{v}(T) u_{T}, D\right), T \in \Omega^{N}$, the null player property implies that $x_{i}=0$ for all $i \in N \backslash T$, and efficiency implies that $\sum_{i \in T} x_{i}=\Delta_{v}(T)$. With nonnegativity it follows that $x_{i} \geq 0$ for all $i \in T$. Since $i, j \in T$ veto each other in $u_{T}$, structural monotonicity implies that $x_{i} \geq x_{j}$ for all $i, j \in T$ with $(i, j) \in D$. Hence, $x \in \Delta_{v}(T) P_{D}^{T}$ and, consequently, $x \in \operatorname{RC}\left(\Delta_{v}(T) u_{T}, D\right)$. The latter inclusion shows that $\mathcal{F}\left(\Delta_{v}(T) u_{T}, D\right) \subseteq \mathrm{RC}\left(\Delta_{v}(T) u_{T}, D\right)$. Finally, additivity of $\mathcal{F}$ and RC implies that $\mathcal{F}(v, D)=\sum_{T \in \Omega^{N}} \mathcal{F}\left(\Delta_{v}(T) u_{T}, D\right) \subseteq \sum_{T \in \Omega^{N}} \operatorname{RC}\left(\Delta_{v}(T) u_{T}, D\right)=$ $\mathrm{RC}(v, D)$ for all $v \in \mathcal{G}_{+}^{N}$ and $D \in \mathcal{D}^{N}$.

Notice that the proofs of the Theorems 5.2 and 5.3 also hold on any subset of digraphs $\mathcal{E}$ of $\mathcal{D}^{N}$, and thus the results hold for any solution on any subclass $\mathcal{G}_{+}^{N} \times \mathcal{E}, \mathcal{E} \subseteq \mathcal{D}^{N}$. So, (i) for any $\mathcal{E} \subseteq \mathcal{D}^{N}$, the Restricted Harsanyi set solution RC satisfies efficiency, the null player property, additivity, nonnegativity and structural monotonicity on $\mathcal{G}_{+}^{N} \times \mathcal{E}$, and (ii) if solution $\mathcal{F}$ on $\mathcal{G}_{+}^{N} \times \mathcal{E}$ satisfies these properties then $\mathcal{F}(v, D) \subseteq \operatorname{RC}(v, D)$ for all $(v, D) \in \mathcal{G}_{+}^{N} \times \mathcal{E}$. In particular this holds when $\mathcal{E}$ is the class of all acyclic directed graphs or the class of all rooted (directed) trees. But also when $\mathcal{E}$ only contains the empty digraph $(N, D)$ with $D=\emptyset$ as its unique element. In that case $\mathcal{G}_{+}^{N} \times \mathcal{E}$ reduces to the class $\mathcal{G}_{+}^{N}$ of totally positive games and, according to (ii) of Theorem $4.4, \mathrm{RC}(v, \emptyset)=$ Core $(v)$ for all $v \in \mathcal{G}_{+}^{N}$. Moreover, in this case the structural monotonicity property gives no restriction, and thus Theorem 5.3 characterizes the Core on the class of totally positive games as the 'maximal' (with respect to set inclusion) solution that satisfies efficiency, the null player property, additivity and nonnegativity. ${ }^{8}$ On the other hand, when $\mathcal{E}$ only contains the complete (irreflexive) digraph $(N, D)$ with $D=D^{c}$ as its unique element,

\footnotetext{
${ }^{8}$ Here we should redefine efficiency, the null player property, additivity and nonnegativity for TU-games by adapting the corresponding axioms for games with ordered players in a straightforward way.
} 
then $\mathrm{RC}\left(v, D^{c}\right)=\{S h(v)\}$ for all $v \in \mathcal{G}_{+}^{N}$, and structural monotonicity requires unanimity symmetry (i.e., in a unanimity game all players of the unanimity coalition earn the same). In this case we obtain a characterization of the Shapley value for totally positive TUgames as the unique solution satisfying efficiency, the null player property, additivity and unanimity symmetry. Notice that in this case the nonnegativity axiom (although satisfied by the Shapley value) is superfluous in the characterization.

Corollary 5.4 On the class $\mathcal{G}_{+}^{N}$ of totally positive TU-games:

(i) the Core solution satisfies efficiency, the null player property, additivity and nonnegativity;

(ii) if solution $F$ on $\mathcal{G}_{+}^{N}$ satisfies efficiency, the null player property, additivity and nonnegativity, then $F(v) \subseteq$ Core $(v)$ for all $v \in \mathcal{G}_{+}^{N}$;

(iii) the Shapley value is the unique single-valued solution on $\mathcal{G}_{+}^{N}$ that satisfies efficiency, the null player property, additivity and unanimity symmetry.

We obtain a full axiomatization of the Restricted Core solution if we add the following three axioms, of which the first two are standard in game theory.

Axiom (Nonemptyness) For all $(v, D) \in \mathcal{G}_{+}^{N} \times \mathcal{D}^{N}$, the set $\mathcal{F}(v, D) \subset \mathbb{R}^{n}$ is not empty.

Axiom (Convexity) For all $(v, D) \in \mathcal{G}_{+}^{N} \times \mathcal{D}^{N}$, the set $\mathcal{F}(v, D) \subset \mathbb{R}^{n}$ is convex.

The last property is a consistency property. First, we define the coalitional proxy game of a coalition $T$ with respect to a subset $R$ of $T$. In this game the coalition $R \subseteq T$ acts as a proxy for $T$ in the sense that the dividend of a coalition $T$ is assigned to its subset $R$ by giving every player in $R$ an equal share in the dividend of $T$. This yields the coalitional proxy game $v_{R, T}$ given by

$$
v_{R, T}(S)=\widetilde{v}(S)+\frac{|R \cap S|}{|R|} \Delta_{v}(T), \quad S \subseteq N,
$$

where $\widetilde{v}=v-\Delta_{v}(T) u_{T}$ is the game with $\widetilde{v}(S)=v(S)-\Delta_{v}(T)$ for every $S$ such that $T \subseteq S$, and $\widetilde{v}(S)=v(S)$ otherwise. Note that $v_{R, T}(N)=v(N)$, and thus any efficient solution distributes the same amount in games $v$ and $v_{R, T}$. The next lemma follows by straightforward calculation of the dividends and is given without proof.

Lemma 5.5 For $T \subseteq N$ and $R \subseteq T$ the dividends of game $v_{R, T}$ are given by

$$
\Delta_{v_{R, T}}(S)= \begin{cases}0 & \text { if } S=T \\ \Delta_{v}(S)+\frac{1}{|R|} \Delta_{v}(T) & \text { if } S=\{j\} \text { and } j \in R \\ \Delta_{v}(S) & \text { otherwise. }\end{cases}
$$


The lemma shows that coalition $T$ is a 'null coalition' in game $v_{R, T}$, in the sense that its dividend is zero, while the dividends of all single players in $R$ are increased with a fraction $\frac{1}{|R|}$ of the dividend of $T$. We now state the coalitional consistency property. Recall that $\mathcal{C}_{D}^{T}$ is the collection of subsets of $T$ that are complete (i.e., connected and comprehensive from above) in the subgraph $(T, D(T))$ of $(N, D)$.

Axiom (Coalitional consistency) For every $(v, D) \in \mathcal{G}_{+}^{N} \times \mathcal{D}^{N}$ and every $R \in \mathcal{C}_{D}^{T}$ with $T \in \Omega^{N}$, it holds that $\mathcal{F}\left(v_{R, T}, D\right) \subseteq \mathcal{F}(v, D)$.

This property ${ }^{9}$ says that by allocating the dividend of a coalition $T$ equally among the players of any complete subset $R$ of that coalition $T$, we do not obtain new payoff vectors in the solution. Going from a game $v$ with ordered players to the coalitional proxy game $v_{R, T}$ with $R \in \mathcal{C}_{D}^{T}$ and the same ordering of the players, we do not yet assign payoffs to the players, but we allocate one of the coalitional dividends to singleton dividends. Considering this as a partial solution, the consistency requirement states that the payoff vectors assigned to the new game should also be payoff vectors in the original game.

To prove that $\operatorname{RC}(v, D)$ is characterized by the eight axioms stated above, we first state three lemmas.

Lemma 5.6 Let $\mathcal{F}$ be a solution on $\mathcal{G}_{+}^{N} \times \mathcal{D}^{N}$ satisfying efficiency, the null player property, nonnegativity and structural monotonicity. Then for any $D \in \mathcal{D}^{N}$ and $T \in \Omega^{N}$, we have that $\mathcal{F}\left(u_{T}, D\right) \subseteq P_{D}^{T}$.

Proof. Let $x \in \mathcal{F}\left(u_{T}, D\right)$. Then, from efficiency it follows that $\sum_{i \in N} x_{i}=1$ and by nonnegativity we have that $x_{i} \geq 0, i \in N$. From the null player property we have that $x_{i}=0$ if $i \in N \backslash T$. Finally, from structural monotonicity we obtain that for any two players $i, j \in T$ with $(i, j) \in D$ it holds that $x_{i} \geq x_{j}$. Hence $\mathcal{F}\left(u_{T}, D\right) \subseteq P_{D}^{T}$.

In the proof of the next lemma we apply Theorem 4.5 on the extreme points of $P_{D}^{T}$.

Lemma 5.7 Let $\mathcal{F}$ be a solution on $\mathcal{G}_{+}^{N} \times \mathcal{D}^{N}$ satisfying nonemptyness, convexity, efficiency, the null player property, additivity and coalitional consistency. Then for any $D \in \mathcal{D}^{N}$ and $T \in \Omega^{N}$, we have that $P_{D}^{T} \subseteq \mathcal{F}\left(u_{T}, D\right)$.

Proof. It follows from Lemma 5.5 that for $R \subseteq T$ the game $w_{R, T}=\left(u_{T}\right)_{R, T}$ satisfies

$$
w_{R, T}=\sum_{i \in R} \frac{1}{|R|} u_{\{i\}} .
$$

\footnotetext{
${ }^{9}$ We remark that this is a different kind of consistency than reduced game consistency which is often used in characterizing solutions for cooperative games.
} 
From the nonemptyness, efficiency and the null player property it follows that for any $D$, $\mathcal{F}\left(\frac{1}{|R|} u_{\{i\}}, D\right)=\left\{\frac{1}{R} a^{\{i\}}\right\}, i \in N$. Additivity implies that $\mathcal{F}\left(w_{R, T}, D\right)=\sum_{i \in R} \mathcal{F}\left(\frac{1}{|R|} u_{\{i\}}, D\right)$ $=\sum_{i \in R}\left\{\frac{1}{|R|} a^{\{i\}}\right\}=\left\{a^{R}\right\}$. Hence coalitional consistency implies that $a^{R} \in \mathcal{F}\left(u_{T}, D\right)$ for any $R \in \mathcal{C}_{D}^{T}$. Finally, with Theorem 4.5, convexity implies that $P_{D}^{T}=\operatorname{Conv}\left(\left\{a^{R}, R \in\right.\right.$ $\left.\left.\mathcal{C}_{D}^{T}\right\}\right) \subseteq \mathcal{F}\left(u_{T}, D\right)$

Lemma 5.8 Let $\mathcal{F}$ be a solution on $\mathcal{G}_{+}^{N} \times \mathcal{D}^{N}$ satisfying nonemptyness, convexity, efficiency, the null player property, additivity, nonnegativity, structural monotonicity and coalitional consistency. Then for any $D \in \mathcal{D}^{N}, T \in \Omega^{N}$ and $c>0$, we have that $\mathcal{F}\left(c u_{T}, D\right)=c P_{D}^{T}$.

Proof. For $c=1$, the statement follows immediately from Lemmas 5.6 and 5.7. For positive $c \neq 1$ it follows from the fact that the set of extreme points of $c P_{D}^{T}$ is given by $\operatorname{Ex}\left(c P_{D}^{T}\right)=c \operatorname{Ex}\left(P_{D}^{T}\right)$. The result then follows by repeating the reasoning given in the Lemmas 5.6 and 5.7 for the games $c u_{T}$.

We now come to a full axiomatization of the Restricted Core.

Theorem 5.9 A solution $\mathcal{F}$ on $\mathcal{G}_{+}^{N} \times \mathcal{D}^{N}$ satisfies nonemptyness, convexity, efficiency, the null player property, additivity, nonnegativity, structural monotonicity and coalitional consistency if and only if $\mathcal{F}$ is the Restricted Core solution.

Proof. It is straightforward to verify that the Restricted Core solution satisfies these properties on $\mathcal{G}_{+}^{N} \times \mathcal{D}^{N}$. To show uniqueness, suppose that a solution $\mathcal{F}$ on $\mathcal{G}_{+}^{N} \times \mathcal{D}^{N}$ satisfies the eight axioms. From additivity and Lemma 5.8 we obtain that $\mathcal{F}(v, D)=$ $\sum_{T \in \Omega^{N}} \mathcal{F}\left(\Delta_{v}(T) u_{T}, D\right)=\sum_{T \in \Omega^{N}} \Delta_{v}(T) P_{D}^{T}=\mathrm{RC}(v, D)$.

We end this section by showing logical independence of the eight axioms in Theorem 5.9 using the following alternative solutions on $\mathcal{G}_{+}^{N} \times \mathcal{D}^{N}$.

1. Solution $\mathcal{F}_{N}$ given by $\mathcal{F}_{N}(v, D)=\emptyset$ satisfies all axioms except nonemptyness.

2. Solution $\mathcal{F}_{C}$ given by $\mathcal{F}_{C}(v, D)=\sum_{T \in \Omega^{N}} \operatorname{Ex}\left(P_{D}^{T}\right)$ satisfies all axioms except convexity.

3. Solution $\mathcal{F}_{E}$ given by $\mathcal{F}_{E}(v, D)=\mu \mathrm{RC}(v, D)$ for some positive $\mu \neq 1$, satisfies all axioms except efficiency.

4. Define $\bar{v} \in \mathbb{R}^{n}$ by $\bar{v}_{i}=\frac{1}{|N|} v(N)$. Solution $\mathcal{F}_{N P}$ given by $\mathcal{F}_{N P}(v, D)=\mu\{\bar{v}\}+$ $(1-\mu) \mathrm{RC}(v, D)$, for some $\mu, 0<\mu<1$, satisfies all axioms except the null player property. 
5. For game $v \in \mathcal{G}^{N}$, define game $\bar{v} \in \mathcal{G}^{N}$ by $\bar{v}=\left(\sum_{T \subseteq N, T \neq \emptyset} \Delta_{v}(T)\right) u_{N(v)}$, where $N(v)$ is the set of non-null players in $v$. Solution $\mathcal{F}_{A}$ given by

$$
\mathcal{F}_{A}(v, D)= \begin{cases}R C(v, D) & \text { if } v(N) \geq 10 \\ R C(\bar{v}, D) & \text { if } v(N)<10\end{cases}
$$

satisfies all axioms except additivity.

6. For some $\epsilon>0$, define $P^{T}(\epsilon)=\left\{p^{T} \in \mathbb{R}^{n} \mid p_{i}^{T}=0\right.$, if $i \notin T, p_{i}^{T} \geq-\epsilon$ if $i \in T$, and $\left.\sum_{i \in T} p_{i}^{T}=1, T \in \Omega^{N}\right\}$, and define the solution $\operatorname{RC}^{\epsilon}$ by $\operatorname{RC}^{\epsilon}(v, D)=$ $\sum_{T \in 2^{N} \backslash\{\emptyset\}} \Delta_{v}(T) P_{D}^{T}(\epsilon)$. Solution $\mathcal{F}_{N N}$ given by $\mathcal{F}_{N N}(v, D)=\operatorname{RC}^{\epsilon}(v, D)$ satisfies all axioms except nonnegativity.

7. Solution $\mathcal{F}_{S M}$ given by $\mathcal{F}_{S M}(v, D)=\operatorname{Core}(v)$ satisfies all axioms except structural monotonicity.

8. Solution $\mathcal{F}_{C C}$ given by $\mathcal{F}_{C C}(v, D)=\{S h(v)\}$ satisfies all axioms except coalitional consistency.

\section{The Restricted Selectope}

The Selectope, introduced by Hammer et al. (1977) is a solution for TU-games that is equivalent to the Harsanyi set, and thus yields an alternative definition of this solution, see also Derks et al. (2000). For totally positive games the selectope thus yields another definition of the Core. First, a selector chooses for every coalition a particular player in the coalition to whom to assign the dividend of that coalition, i.e., a selector is a function $\alpha: \Omega^{N} \rightarrow N$ such that $\alpha(T) \in T$ for all $T \subseteq N$. The selectope vector corresponding to selector $\alpha$ and game $v \in \mathcal{G}^{N}$ is the vector $s^{\alpha}(v) \in \mathbb{R}^{n}$ given by $s_{i}^{\alpha}(v)=\sum_{\left\{T \in \Omega^{N} \mid \alpha(T)=i\right\}} \Delta_{v}(T)$ and assigns the dividend of any nonempty coalition $T$ fully to the player $\alpha(T)$ from this coalition that is selected by the selector $\alpha$. The Selectope $S(v)$ of $v$ then is the convex hull of all selectope vectors, i.e.,

$$
S(v)=\operatorname{Conv}\left(\left\{s^{\alpha}(v) \mid \alpha \in \mathcal{A}^{N}\right\}\right),
$$

with $\mathcal{A}^{N}=\left\{\alpha: \Omega^{N} \rightarrow N \mid \alpha(T) \in T\right.$ for all $\left.T \in \Omega^{N}\right\}$ being the set of all selectors on $N$. Now, if the players are ordered according to digraph $D \in \mathcal{D}^{N}$ then we put as a restriction on a selector that the dividend of a coalition should always be assigned to a player that has no predecessors in the coalition. Formally this is done by considering only selectors in the set

$$
\mathcal{A}_{D}^{N}=\left\{\alpha \in \mathcal{A}^{N} \mid \text { for every } T \in \Omega^{N}:[\{i, j\} \subseteq T \text { and }(i, j) \in D] \Rightarrow \alpha(T) \neq j\right\} .
$$

We obtain the following definition. 
Definition 6.1 The Restricted Selectope of game with ordered players $(v, D) \in \mathcal{G}_{+}^{N} \times \mathcal{D}^{N}$ is the set $\mathcal{S}(v, D)=\operatorname{Conv}\left(\left\{s^{\alpha}(v) \mid \alpha \in \mathcal{A}_{D}^{N}\right\}\right)$.

We refer to the solution that assigns to every game with ordered players its Restricted Selectope as the Restricted Selectope solution. Obviously, for every $(v, D) \in \mathcal{G}_{+}^{N} \times \mathcal{D}^{N}$ we have $\mathcal{S}(v, D) \subseteq S(v)$ since $\mathcal{A}_{D}^{N} \subseteq \mathcal{A}^{N}$. Note that $\mathcal{A}_{D}^{N} \neq \emptyset$ if and only if there is no cycle in $D$. Moreover, $\mathcal{A}_{D}^{N}=\mathcal{A}^{N}$ if and only if $D=\emptyset$. Therefore, $\mathcal{S}(v, D)=\emptyset$ if there is a cycle in $D$, and $\mathcal{S}(v, D)=\operatorname{Core}(v)$ if $D=\emptyset$.

Example 6.2 The Restricted Selectope of the game with ordered players $(v, D)$ of Example 4.2 is given by $\mathcal{S}(v, D)=\operatorname{Conv}\left(\left\{(2,0,0)^{\top},(1,1,0)^{\top}\right\}\right)$.

We consider $\operatorname{RC}(v, D)$ to contain more 'fair' payoff vectors than $\mathcal{S}(v, D)$. For example, in the game $(v, D)$ of Examples 4.2 and 6.2 it seems to be too tough on player 3 to exclude him from any positive dividend, as is the case in $\mathcal{S}(v, D)$. However, the position of the players in the digraph $D$ should be reflected in the payoff distributions, which is the case in $\mathrm{RC}(v, D)$. The reason to discuss the Restricted Selectope here is that one encounters it implicitly in several economic applications, as we discuss later.

Although the Core and Selectope are equivalent for totally positive TU-games, i.e., Core $(v)=H(v)=S(v)$ for every $v \in \mathcal{G}_{+}^{N}$, the Restricted Core and Restricted Selectope are different. Clearly, on the class of totally positive games with ordered players also the Restricted Selectope is a refinement of the Core, but can be empty. The Restricted Selectope solution satisfies efficiency, the null player property, additivity, nonnegativity and structural monotonicity. By Theorem 5.3 it then follows that the Restricted Selectope is always a subset of the Restricted Core ${ }^{10}$.

Corollary 6.3 For every $(v, D) \in \mathcal{G}_{+}^{N} \times \mathcal{D}^{N}$ it holds that $\mathcal{S}(v, D) \subseteq R C(v, D)$.

The Restricted Selectope solution also satisfies an alternative structural monotonicity property which requires that a vetoed player earns zero payoff.

Axiom (Strict structural monotonicity) For all $(v, D) \in \mathcal{G}_{+}^{N} \times \mathcal{D}^{N}$ and $j \in N$, any payoff vector $x \in \mathcal{F}(v, D)$ satisfies $x_{j}=0$ whenever there is some $i \in N \backslash\{j\}$ such that both $(i, j) \in D$ and $j$ is vetoed by $i$ in $v$.

A similar requirement for simple TU-games is discussed by Napel and Widgrén (2001). Note that the structural monotonicity property and the strict structural monotonicity property do not imply one another. Replacing in Theorems 5.2 and 5.3 the structural

\footnotetext{
${ }^{10}$ Note that it is also straightforward to give a direct proof of this.
} 
monotonicity property by the strict structural monotonicity property, we obtain characterizations of the Restricted Selectope solution on the class $\mathcal{G}_{+}^{N} \times \mathcal{D}^{N}$ of totally positive games with ordered players.

Theorem 6.4 (i) The Restricted Selectope solution $\mathcal{S}$ satisfies efficiency, the null player property, additivity, nonnegativity and strict structural monotonicity on $\mathcal{G}_{+}^{N} \times \mathcal{D}^{N}$.

(ii) If solution $\mathcal{F}$ on $\mathcal{G}_{+}^{N} \times \mathcal{D}^{N}$ satisfies efficiency, the null player property, additivity, nonnegativity and strict structural monotonicity, then $\mathcal{F}(v, D) \subseteq \mathcal{S}(v, D)$ for all $(v, D) \in$ $\mathcal{G}_{+}^{N} \times \mathcal{D}^{N}$

Proof. (i) The Restricted Selectope solution $\mathcal{S}$ satisfying efficiency, the null player property, additivity and nonnegativity follows similar as for the Harsanyi set $H$ for TU-games in $\mathcal{G}^{N}$ in Vasil'ev and van der Laan (2002). Further it follows straightforward that $\mathcal{S}$ satisfies strict structural monotonicity.

(ii) Suppose that solution $\mathcal{F}$ on $\mathcal{G}_{+}^{N} \times \mathcal{D}^{N}$ satisfies the five properties. For $x \in \mathcal{F}\left(\Delta_{v}(T) u_{T}, D\right)$, $T \in \Omega^{N}$, the null player property implies that $x_{i}=0$ for all $i \in N \backslash T$, and efficiency implies that $\sum_{i \in T} x_{i}=\Delta_{v}(T)$. With nonnegativity it follows that $x_{i} \geq 0$ for all $i \in T$. Strict structural monotonicity implies that $x_{j}=0$ for all $i, j \in T$ with $(i, j) \in D$ since $\Delta_{\Delta_{v}(T) u_{T}}(H)=0$ for all $H \neq T$. Hence $x \in \mathcal{S}\left(\Delta_{v}(T) u_{T}, D\right)$, showing that $\mathcal{F}\left(\Delta_{v}(T) u_{T}, D\right) \subseteq \mathcal{S}\left(\Delta_{v}(T) u_{T}, D\right)$. Additivity of $\mathcal{F}$ and $\mathcal{S}$ then implies that $\mathcal{F}(v, D) \subseteq \mathcal{S}(v, D)$ for all $(v, D) \in \mathcal{G}_{+}^{N} \times \mathcal{D}^{N}$.

Recall that $\mathcal{S}(v, D)=\emptyset$ if $D$ is cyclic. Considering solutions $\mathcal{F}(., D)$ on $\mathcal{G}_{+}^{N}$ for a given specific digraph $D \in \mathcal{D}^{N}$, part (ii) of Theorem 6.4 thus gives as a corollary that in that case there is no nonempty solution satisfying the given properties.

Corollary 6.5 If $D \in \mathcal{D}^{N}$ is cyclic then the only solution $\mathcal{F}(., D)$ on $\mathcal{G}_{+}^{N}$ that satisfies efficiency, the null player property, additivity, nonnegativity and strict structural monotonicity is the empty solution $\mathcal{F}(v, D)=\emptyset$ for all $v \in \mathcal{G}_{+}^{N}$.

\section{The water distribution problem reconsidered}

Applying the Restricted Core to the totally positive game with ordered players $(v, D)$ associated with a water distribution problem as described in Section 3, we obtain exactly the set of allocations described at the end of that section. We illustrate this with an example.

Example 7.1 Consider the water distribution problem among players $N=\{1,2,3\}$, such that $e_{1}=1, e_{2}=e_{3}=0, b^{1}(x)=0, b^{2}(x)=0$ and $b^{3}(x)=x$ for all $x \geq 0$. So, the only water inflow is at the most upstream player 1 , while the most downstream player 3 is the 
only one that obtains a positive benefit from water consumption. Player 2 has neither a water inflow nor positive benefit from water consumption, but is needed to let water pass from player 1 to player 3. The corresponding river game is the unanimity game of the 'grand' coalition given by $v(N)=1$, and $v(S)=0$ otherwise. The Core is the full set of efficient nonnegative payoff vectors $\operatorname{Core}(v)=\left\{z \in \mathbb{R}_{+}^{n} \mid \sum_{i \in N} z_{i}=1\right\}$.

The solution $m^{u}(v)$ of Ambec and Sprumont (2002) assigns the worth $v(N)$ fully to the most downstream player 3, while players 1 and 2 get nothing for letting the water pass through to player 3 . This coincides with the unique element in the Restricted Selectope where the ordering of the players is from downstream to upstream reflected by the digraph $D^{-}=\{(3,2),(2,1)\}$. The payoff vector $m^{u}(v)$ is also an extreme point of the corresponding Restricted Core. Since $\Delta_{v}(N)=1$ and $\Delta_{v}(S)=0$ otherwise, the Restricted Core is given by $\operatorname{RC}\left(v, D^{-}\right)=P_{D^{-}}^{N}=\left\{p \in P^{N} \mid p_{3} \geq p_{2} \geq p_{1}\right\}$. By applying Theorem 4.5 we obtain that $\operatorname{RC}\left(v, D^{-}\right)=\operatorname{Conv}\left(\left\{(0,0,1)^{\top},\left(0, \frac{1}{2}, \frac{1}{2}\right)^{\top},\left(\frac{1}{3}, \frac{1}{3}, \frac{1}{3}\right)^{\top}\right\}\right)$.

On the other hand, the Restricted Core on the more natural ordering from upstream to downstream, reflected by the digraph $D=\{(1,2),(2,3)\}$, is given by $\operatorname{RC}(v, D)=P_{D}^{N}=$ $\left\{p \in P^{N} \mid p_{1} \geq p_{2} \geq p_{3}\right\}=\operatorname{Conv}\left(\left\{(1,0,0)^{\top},\left(\frac{1}{2}, \frac{1}{2}, 0\right)^{\top},\left(\frac{1}{3}, \frac{1}{3}, \frac{1}{3}\right)^{\top}\right\}\right)$, see Figure 2. This is also a subset of the Core with three extreme points. One of these extreme points is the allocation $m^{l}(v)$ assigning the dividend fully to the most upstream player 1 . This is also the unique element in the corresponding Restricted Selectope.

Note that the Shapley value, which distributes the worth equally among the three players, is an extreme point of the Restricted Core corresponding to both orderings.

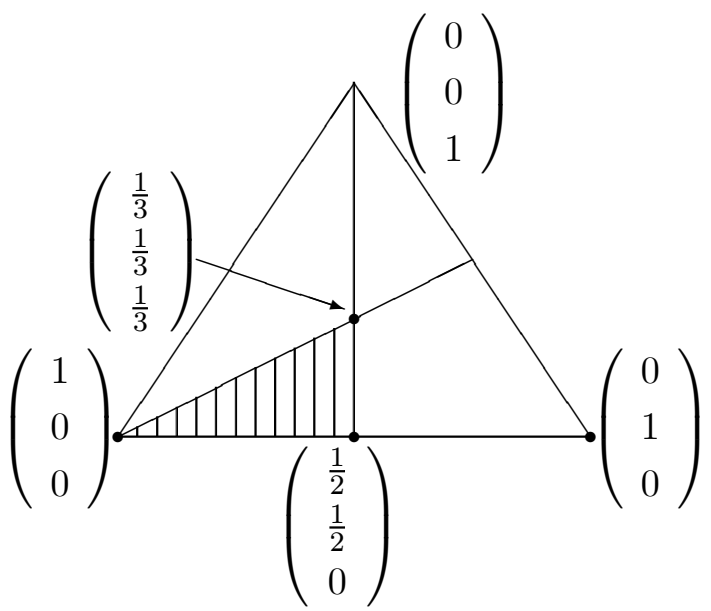

Figure 2: $\mathrm{RC}(v, D)$ of Example 7.1

We want to stress that our critique on the solution $m^{u}$ of Ambec and Sprumont (2002) is the modeling, i.e., ordering the players from downstream to upstream. As shown in the example 
above, the Restricted Core can be applied to this ordering (yielding the solution $m^{u}$ as one of its extreme points), but also to the reverse ordering from upstream to downstream, yielding the marginal value vector $m^{l}$ as one of its extreme points. For any ordering the Shapley value always is an extreme point of the Restricted Core.

\section{Final remarks}

In this paper we assumed that there is an ordering on the players in a totally positive cooperative TU-game. This ordering restricts the payoff distribution in the game and we propose the concept of the Restricted Core as solution concept for such situations. This Restricted Core provides a collection of Core payoff distributions obtained by taking into account the ordering when distributing the dividends. Theorem 4.3 states that the Restricted Core is a subset of the Core of the unrestricted game which always contains the Shapley value of the unrestricted game. We also provided axiomatic characterizations. As a further refinement we discussed the Restricted Selectope which assigns to any totally positive game with ordered players a subset of the Restricted Core, but is empty if the ordering contains a cycle.

Many economic applications are modelled by totally positive TU-games such as sequencing games (see, e.g. Curiel et al. (1989)), auction games (see Graham et al. (1990)), airport games (see Littlechild and Owen (1973)), telecommunication games (see van den Nouweland et al. (1996)) and queueing games (see Maniquet (2003)). We gave special attention to river games arising from water distribution problems. We saw that the solution proposed by Ambec and Sprumont (2002) is the unique element in the Restricted Selectope taking the ordering from downstream to upstream, and is an extreme point of the corresponding Restricted Core. As an alternative we suggested the Restricted Core corresponding to the ordering of the players from upstream to downstream.

In a similar way, one can obtain the hierarchical outcomes for (communication) graph restricted games as introduced in Demange (2004). For communication graph games, Myerson (1977) introduced the restricted game where to every coalition one assigns the sum of the worths of all its components (i.e. maximally connected subsets) in the graph ${ }^{11}$. Given a cycle free undirected communication graph Demange (2004) associates with every node a directed tree obtained by choosing that node as the root, and for each of these directed trees considers the marginal value vector of the restricted game corresponding to orders where 'lower ranked' players enter before 'higher ranked' players. For every

\footnotetext{
${ }^{11}$ In these games with limited communication (graph) structure as introduced by Myerson (1977) the edges of an undirected graph on the set of players represent binary communication links between the players such that players can cooperate only if they are connected, see also e.g. Kalai et al. (1978), Owen (1986) and Borm et al. (1992).
} 
directed (rooted) tree this hierarchical outcome is the unique element of the corresponding Restricted Selectope corresponding to this tree and the restricted game.

Another game theoretic model in which players in a TU-game belong to a hierarchical structure are the games with a permission structure as considered in Gilles et al. (1992), Gilles and Owen (1994), van den Brink and Gilles (1996) and van den Brink (1997) ${ }^{12}$. In these games it is assumed that players need permission from other players before they are allowed to cooperate within a coalition. So, instead of restricting payoff distributions, in games with a permission structure the cooperation possibilities are restricted ${ }^{13}$. Given the sets of feasible coalitions, a restricted game is defined which assigns to every coalition the worth of its largest feasible subset. We want to stress that the approaches of restricted cooperation (as in games with a permission structure) and restricted payoff distribution (as done in the underlying paper) are essentially different. For totally positive games, the Restricted Core is a subset of the Core of the unrestricted game and therefore refines the concept of Core. In the models of restricted cooperation possibilities, the Core of the restricted game is larger than the Core of the original game.

\section{References}

Ambec, S., and Y. Sprumont (2002), Sharing a river, Journal of Economic Theory 107, 453-462.

Aumann R.J., and J.H. Drèze (1974), Cooperative games with coalition structure, International Journal of Game Theory 3, 217-237.

Bilbao, J.M., (1998) Values and Potential of games with Cooperation Structure, International Journal of Game Theory 27, 131-145.

Bondareva, O. (1962), The theory of the core in an $n$-person game, Vestnik Leningrad. Univ. 13, 141-142 (in Russian).

Borm, P., Owen, G., and Tijs, S. (1992), On the position value for communication situations, SIAM Journal of Discrete Mathematics 5, 305-320.

\footnotetext{
${ }^{12}$ Related is also the model of Faigle and Kern (1992) who consider feasible rankings of the players.

${ }^{13}$ In this sense these models fall within the theory on restricted cooperation, together with, for example, the before mentioned games with limited communication (graph) structure of Myerson (1977), and the games in a-prioiri coalition structure in which it is assumed that the set of players is partitioned into disjoint sets which represent social groups such that for a particular player it is more easy to cooperate with players in its own group than to cooperate with players in other groups, see e.g., Aumann and Drèze (1974), Owen (1977), Hart and Kurz (1983) and Winter (1989).
} 
Brink, R. van den (1997), An axiomatization of the disjunctive permission value for games with a permission structure, International Journal of Game Theory 26, 27-43.

Brink, R. van den, and Gilles, R. P. (1996), Axiomatizations of the conjunctive permission value for games with permission structures, Games and Economic Behavior 12, 113126.

Brink, R. van den, G. van der Laan, and V. Vasilev (2006), Distributing dividends in games with ordered players", TI-Discussion Paper 06/114-1, Tinbergen Institute and Free University, Amsterdam.

Brink, R. van den, G. van der Laan, and V.A. Vasil'ev (2007), Component efficient solutions in line-graph games with applications, Economic Theory 33, 349-364.

Brink, R. van den, G. van der Laan, and V.A. Vasil'ev (2008), On the extreme points of two polytopes associated with a digraph and applications to cooperative games, Journal of Mathematical Economics 44, 1114-1125.

Curiel, I., G. Pederzoli, and S. Tijs (1989), Sequencing games, European Journal of Operational Research 40, 344-351.

Demange, G. (2004), On group stability in hierarchies and networks", Journal of Political Economy, 112, 754-778.

Derks, J., H. Haller, and H. Peters (2000), The selectope for cooperative TU-games, International Journal of Game Theory 29, 23-38.

Derks, J., G. van der Laan, and V. Vasil'ev (2006), Characterizations of random order values by Harsanyi payoff vectors, Mathematical Methods of Operations Research 64, 155-164.

Faigle, U., and Kern, W. (1993), The Shapley value for cooperative games under precedence constraints, International Journal of Game Theory 21, 249-266.

Fernández, C., P. Borm, R. Hendrickx and S. Tijs (2005), Drop-out Monotonicity Rules for Sequencing Games, Mathematical Methods of Operations research 61, 501 - 504.

Gilles, R. P., and Owen, G. (1994), Cooperative games and disjunctive permission structures, Department of Economics, Virginia Polytechnic Institute and State University, Blacksburg, Virginia.

Gilles, R. P., Owen, G., and Brink, R. van den (1992), Games with permission structures: the conjunctive approach, International Journal of Game Theory 20, 277-293. 
Gillies, D.B. (1953), Some Theorems on n-Person Games, Princeton University Press, Princeton, NJ.

Graham, D.A., R.C. Marshall, and J-F. Richard (1990), Differential Payments within a Bidder Coalition and the Shapley Value, American Economic Review 80, 493-510.

Greenberg, J. and S. Weber (1986), "Strong Tiebout Equilibrium under Restricted Preferences Domain", Journal of Economic Theory, 38, 101-117.

Hammer, P.L., U.N. Peled, and S. Sorensen (1977), Pseudo-Boolean functions and game theory. I. Core Elements and Shapley Value, Cahiers du CERO 19, 159-176.

Harsanyi, J.C. (1959), A bargaining model for cooperative n-person games, in: Contributions to the Theory of Games IV (eds. Tucker A.W., and R.D. Luce), Princeton UP, Princeton, 325-355.

Hart S., and M. Kurz (1983), Endogeneous Formation of Coalitions, Econometrica 51, 1047-1064.

Ichiishi, T. (1981), Super modularity: applications to convex games and to the greedy algorithm for LP, Journal of Economic Theory 25, 283-286.

Kalai E., A. Postlewaite, and J. Roberts (1978), Barriers to trade and disadvantageous middlemen: nonmonotonicity of the core, Journal of Economic Theory 19, 200-209.

Littlechild, S.C, and G. Owen (1973), A simple expression for the Shapley value in a special case, Management Science 20, 370-372.

Maniquet, F. (2003), A Characterization of the Shapley Value in Queueing Problems, Journal of Economic Theory 109, 90-103.

Myerson, R. B. (1977), Graphs and cooperation in games, Mathematics of Operations Research 2, 225-229.

Nouweland, A. van den, Borm, P., van Golstein Brouwers, W., Groot Bruinderink, R., and S. Tijs (1996), A game theoretic approach to problems in telecommunication, Management Science, 42, 294-303.

Owen, G. (1977), Values of games with a priori unions, in: Essays in Mathematical Economics and Game Theory (eds. Henn R., and O. Moeschlin), Springer Verlag, Berlin, 76-88.

Owen, G. (1986), Values of graph-restricted games, SIAM Journal on Algebraic and Discrete Methods 7, 210-220. 
Shapley, L. S. (1953), A value for N-person games, in: Contributions to the Theory of Games, Vol II (eds. H. W. Kuhn, and A. W. Tucker), Princeton University Press, Princeton, 307-317.

Shapley, L.S. (1967), On balanced sets and cores, Naval Research Logistics Quarterly 14, 453-460.

Shapley, L.S. (1971), Cores of convex games, International Journal of Game Theory 1, 11-26.

Vasil'ev, V.A. (1975), The Shapley value for cooperative games of bounded polynomial variation, Optimizacija Vyp 17, 5-27 (in Russian).

Vasil'ev, V.A. (1978), Support function of the core of a convex game, Optimizacija Vyp 21, 30-35 (in Russian).

Vasil'ev, V.A. (1981), On a class of imputations in cooperative games, Soviet. Math. Dokl. 23, 53-57.

Vasil'ev, V.A. (2006), Cores and generalized NM-solutions for some classes of cooperative games, in: Russian Contributions to Game Theory and Equilibrium Theory (eds. T.S.H. Driessen, G. van der Laan, V.A.Vasil'ev and E.B Yanovskaya), Springer Verlag, Berlin-Heidelberg, 91-149.

Vasil'ev, V.A., and G. van der Laan, (2002), The Harsanyi set for cooperative TU-games, Siberian Advances in Mathematics 12, 97-125.

Weber, R.J. (1988), Probabilistic values for games, in: The Shapley Vakue, Essays in Honor of L.S. Shapley (ed. A.E. Roth), Cambridge University Press, Cambridge, 101-119.

Winter, E. (1989), A value for cooperative games with levels structure of cooperation, International Journal of Game Theory 18, 227-240. 